\title{
Relationship between information technology functionalities and hospital-acquired injurious fall rates in US acute care hospitals
}

\author{
Huey-Ming Tzeng ${ }^{1 *}$, Hsou Mei Hu ${ }^{2}$, Chang-Yi Yin ${ }^{3}$, Yu Kang ${ }^{4}$ \\ ${ }^{1}$ College of Nursing, Washington State University, Spokane, USA \\ ${ }^{2}$ Office of Clinical Affairs, University of Michigan Hospitals \& Health Centers, Ann Arbor, USA \\ ${ }^{3}$ Department of History, Chinese Culture University, Taipei, Chinese Taipei \\ ${ }^{4}$ Department of Public Health and Health Sciences, School of Health Professions and Studies, University of Michigan-Flint, Flint, \\ USA \\ Email: "
}

Received 29 January 2012; revised 13 February 2012; accepted 22 March 2012

\begin{abstract}
The purpose of this exploratory study was to determine health information technology functionalities in inpatient care units that were associated with reduced fall risk among adult patients aged 65 years or older in acute care hospitals in the United States. This study compared the differences in the hospital-acquired injurious fall rates for hospitals in California, Florida, and New York with and without fully implemented IT functionalities in their general medical and surgical inpatient units. It used publicly available 2007 datasets, the hospital was the unit of analysis, and teaching and non-teaching hospitals were analyzed separately. Hospital-acquired injurious falls were identified based on fall-related primary and secondary diagnoses and were flagged by the hospitals as not "present on admission" in the 2007 California, Florida, and New York State Inpatient Database data. The 4 health IT functionalities in general medical and surgical inpatient units were 1) electronic clinical documentation; 2) results viewing; 3 ) computerized provider order entry; and 4) decision support. The research question was What are the effective health IT functionalities in the general medical and surgical units for reducing fall risk among adult patients aged 65 years or older at their hospitals? Independent $t$ tests were used. The results showed that no significant difference was found in the hospital-acquired injurious fall rates between hospitals with and without each of the 4 functionalities and between the teaching hospitals with and without each of the 4 functionalities. Significant differences were found in the injurious fall rates between non-teaching hospitals with and without electronic clinical docu-
\end{abstract}

\footnotetext{
"Corresponding author.
}

mentation and result viewing. Future research may focus on assessing the clinicians' use of the IT functionalities of electronic clinical documentation and results viewing, as well as the effect of the clinicians' use patterns on patient outcomes.

Keywords: Hospitals; Accidental Fall; Inpatients; Electronic Health Records; Clinical Decision Support Systems; Order Entry Systems; Safety; Quality of Health Care

\section{INTRODUCTION}

Hospital-acquired fall-related injuries are still a widespread concern and a challenging patient safety issue in hospitals settings. The systematic review study conducted by Oliver and associates [1] concluded that the most appropriate approach to fall prevention in the hospital environment was multifactorial interventions with multiprofessional input. This far, limited evidence was available to support the effectiveness of single interventions (e.g., delirium avoidance programs, reducing sedative and hypnotic medication, and patient education). Recently, the Agency for Healthcare Research and Quality established hospital-related long-term health information technology (IT) portfolio outcome goals to reduce medical errors and improve patient safety [2]. However, to date, the contribution of health IT to reducing injurious falls in acute care hospitals has not been systematically studied [3] and the limited evidence was inconclusive [4]. For example, the study conducted by Tzeng and associates [4] found that adopting the computerized systems of the decision support systems for drug allergy alerts, drug-drug interaction alerts, and drug-laboratory interaction alerts, were associated with lower injurious fall rates. 


\section{Study Purpose}

The purpose of this exploratory study was to determine health information technology functionalities in inpatient care units that were associated with reduced fall risk among adult patients aged 65 years or older in acute care hospitals in the United States. We proposed having fully implemented health IT functionalities in general medical and surgical inpatient units as structure measures. This study compared the differences in the hospital-acquired injurious fall rates for hospitals in California, Florida, and New York with and without fully implemented IT functionalities in their general medical and surgical inpatient units. It used 3 publicly available 2007 datasets, the hospital was the unit of analysis, and teaching and nonteaching hospitals were analyzed separately.

Hospital-acquired injurious falls were identified based on fall-related primary and secondary diagnoses and were flagged by the hospitals as not "present on admission" (PoA) in the 2007 California, Florida, and New York State Inpatient Database (SID) data $[5,6]$. The 4 health IT functionalities in general medical and surgical inpatient units were 1) electronic clinical documentation; 2) results viewing; 3 ) computerized provider order entry (CPOE), and 4) decision support [7,8].

The research question was What are the effective health IT functionalities in the general medical and surgical units for reducing fall risk among adult patients aged 65 years or older at their hospitals?

To date, limited reports on the effectiveness of IT functionalities has been published to inform practitioners of best practice interventions to reduce fall risk in general medical and surgical units [4]. Our findings could provide information to hospital administrators for quality improvement efforts related to adopting effective computerized systems that can lead to better interventions to reduce fall risk in teaching and non-teaching hospitals.

\section{BACKGROUND}

\subsection{Financial Incentives to Reduce Hospital-Acquired Injuries}

In the United States in October 2007, Medicare began requiring hospitals to include the PoA indicator on all Medicare claims they submit. This requirement has allowed distinguishing inpatient complications that are hospital-acquired from those present before admission [5]. As part of the Medicare Value-Based Purchasing program, PoA indicator reporting have been used to target hospital-acquired conditions (HACs), including hospital-acquired fracture, dislocation, or intracranial injuries due to falls.

Since October 2008, Medicare has no longer reimbursed acute care hospitals for the additional care required due to hospital-acquired injuries (e.g., injurious falls). Hospitals are paid for an episode of inpatient care with HACs minus the HAC-associated costs [5]. In addition, the recent U.S. health care bill, the 2010 Patient Protection and Affordable Care Act, imposed an 1\% penalty on Medicare payments to hospitals in the top quartile of HAC rates that will begin in 2015 [9]. Because of these financial pressures, hospitals are searching for effective practices to prevent hospital-acquired injurious falls.

\subsection{Promotion of Health IT Solutions in US Hospitals}

As stated in the report by Stead and Herbert [10], electronic health information is meant to provide adequate structure and cognitive support to help clinicians avoid mistakes. In the United States, the 4 hospital-related long-term health IT portfolio outcome goals are 1) successful deployment of hospital health IT systems that reduce medical errors and improve patient safety, 2) increased identification and reporting of medical errors and adverse events, 3 ) decreased number of actual errors and adverse events, and 4) increased number of hospitals using electronic health records (EHRs) and CPOE with clinical decision support systems [2]. Since 2003, several initiatives have been introduced to promote our knowledge and understanding of the value of health IT toward improving patient safety and quality of care [2]. Research on the value of health IT for improving patient safety and preventing adverse events (e.g., hospital-acquired injurious falls) is still desperately needed.

The Health IT for Economic and Clinical Health (HITECH) Act in the United States sets goals to promote IT uses for medical practices that are more evidence-based, efficient and less error-prone [11]. Beginning in 2011, health-care providers will be offered financial incentives for demonstrating meaningful use of EHRs. The HITECH Act has authorized incentive payments through federal health insurance programs to speed the adoption of EHRs that show meaningful use. Incentives from the federal government will be offered until 2015 [11].

The study conducted by Jha and associates [12] found that $1.5 \%$ of US hospitals have a comprehensive EHR, and $17 \%$ have implemented $\mathrm{CPOE}$ for medications. Teaching hospitals were likely to have EHR systems. However, it is unknown whether fully implemented health IT systems are linked to expected clinical outcomes (e.g., lower prevalence of the hospital-acquired injurious falls) of a particular hospital and whether the impact of health IT on the quality of care vary in teaching and non-teaching hospitals.

\subsection{Hospital-Acquired Injurious Falls as a Targeted Patient Outcome}

The National Quality Forum (NQF) [13] in the United 
States has suggested outcome, process, structure, and patient-centered measures as considerations for supporting quality improvement efforts in hospitals. For fall and injurious fall assessment, one of the structure measures is the presence of measurable structures to ensure accountability for performance. Inspired by the reports by the NQF and Stead and Herbert [10], we propose in this current study that fully implemented health IT functionalities in general medical and surgical inpatient units can be used as measurable structure measures. The 4 healthrelated IT functionalities in general medical and surgical inpatient units were 1) electronic clinical documentation, 2) results viewing, 3) CPOE, and 4) decision support, as defined by the AHA IT survey supplement $[7,8]$. The hospital-acquired injurious fall rate was a measurable health-care quality improvement indicator.

\subsection{Theoretical Framework}

This study was designed based on the suggestions of the NQF [13] that outcome, process, structure, and patient-centered measures should be considered for supporting a hospital's quality improvement. We proposed that the hospital-acquired injurious fall rate was a measurable health-care quality improvement indicator. Having fully implemented health IT functionalities in each hospital's general medical and surgical inpatient units was conceptualized as a measurable structure measure. Thus far, no theoretical framework is available to guide the analyses, nor do literatures exist to pinpoint potentially effective IT functionalities that may allow clinicians to improve their practice decisions and decrease the occurrences and prevalence of hospital-acquired injurious falls.

As an exploratory study, this work included the 4 IT functionalities as defined in the AHA IT survey supplement $[7,8]$. Based on the findings in the study conducted by Jha and associates [12] that teaching hospitals were more likely to have EHR systems than non-teaching hospitals, teaching status was included in the analyses. This exploratory study included 4 research hypotheses which were:

1) If a hospital has fully implemented the electronic clinical documentation system in its medical/surgical units, the hospital-acquired injurious fall rates will be lower.

2) If a hospital has fully implemented the result viewing system in its medical/surgical units, the hospital-acquired injurious fall rates will be lower.

3) If a hospital has fully implemented the CPOE system in its medical/surgical units, the hospital-acquired injurious fall rates will be lower.

4) If a hospital has fully implemented the decision support system in its medical/surgical units, the hospi- tal-acquired injurious fall rates will be lower.

\section{METHODS}

\subsection{Design}

This exploratory study used 3 publicly available large datasets for analyses. It was approved by the Institutional Review Board of the employed university. The unit of analysis was the hospital. California, Florida, and New York SID data $[5,6]$ beginning on January 1 and ending on December 31, 2007 were used.

Only 3 states were included in the analysis due to a limited budget for purchasing and processing SID data. These states were chosen because the SID data with the PoA indicator for analyses have been used in previous studies $[14,15]$, indicating accepted reliability and validity of the data. Additionally, the SID data for Florida and New York were included because of their geographical locations for comparison purposes. The California and New York have required PoA flags to be coded for diagnoses at discharge since 2002. Further, the California and New York SID data have been used in developing patient safety indicators [14]. We included the Florida SID data because of the state's high spending for its Medicare population [16].

\subsection{Inclusion and Exclusion Criteria}

The inclusion criterion was that patients had been discharged from non-federal, general acute care hospitals (i.e., non-federal government, non-governmental and not-for-profit, and investor-owned for profit hospitals) located in California, Florida, and New York, as defined in the SID. This study included only general acute care hospitals that are members of the American Hospital Association (AHA), had provided diagnostic and therapeutic patient services for a variety of medical conditions [17], and had completed the 2007 AHA Annual IT survey supplement. Patients discharged from hospitals not accessible to the general public were excluded. Only the hospitals with valid information from both SID and the 2007 AHA Annual IT survey supplement data were included in the analysis.

\subsection{Data Sources}

\subsubsection{SID [6]}

Hospital-acquired injurious falls were identified based on fall-related primary and secondary diagnoses (ICD-9 codes within the ranges of $800-829,830-839,850-$ 854 ) and were flagged as not PoA. Injurious falls with a simple, complicating, or major complicating condition were all included. The injurious fall rate among patients aged 65 years or older was calculated as the following: 
(the number of total hospital-acquired injurious falls that occurred among patients aged 65 years or older/total discharges of patients 65 years or older) $\times 1000$ discharges.

\subsubsection{AHA Annual Survey Database [17]}

Teaching status was abstracted from the AHA Annual Survey database. Teaching hospitals were defined based on 2 items in the survey: having residency training approval by accreditation from the Council for Graduate Medical Education ( $1=$ yes) or had medical school affiliation reported to the American Medical Association (1 = yes). Based on the responses for these 2 items, teaching status was coded as $1=$ yes or $0=$ no.

\subsubsection{AHA Hospital Information Technology Supplement Database [7,8]}

Four computerized functionalities were included in the IT survey supplement, under the main question Are the functionalities listed below fully implemented in the following locations? and the subheading of General medical/surgical wards. These 4 functionalities were 1) electronic clinical documentation, 2) results viewing, 3) CPOE, and 4) decision support. All 4 items were operationalized by the IT survey supplement and measured as $1=$ yes (fully implemented) or $2=$ no (including partial and not yet implemented as defined by AHA). The scale used in the IT survey supplement was used in this study without further recoding.

\subsection{Data Management}

The hospital-level data of the 3 data sources were merged into one single dataset using SPSS 17.0 Window version (Chicago, IL, USA). Data were matched based on each hospital's Medicare provider ID. Medicare provider ID was the only overlapping information across data sources.

\subsection{Statistical Analysis}

SPSS 17.0 Windows version was used. Descriptive analyses were used to describe study variables. Independent $t$ tests were used to answer the research question and test the research hypotheses, as well as to compare the differences between the hospitals with and without each 1 of 4 fully implemented IT functionalities in their general medical and surgical inpatient units, on the hospital-acquired injurious fall rates at acute care hospitals. The analyses were conducted separately on teaching and non-teaching hospitals. To increase the sample size, hospitals in California, Florida, and New York were analyzed at the same time. For statistical analyses, alpha was set at 0.05 .

\section{RESULTS}

\subsection{Descriptive Analyses}

Among 284 hospitals included in the study, 129 were in California, 74 in Florida, and 81 in New York. A total of $195(68.7 \%)$ hospitals were non-teaching facilities and $89(31.3 \%)$ were teaching facilities. Seven hospitals $(2.5 \%)$ were investor-owned with $<100$ beds, $45(15.8 \%)$ were investor-owned with $\geq 100$ beds, 19 (6.7\%) were non-profit, rural with $<100$ beds, $21(7.4 \%)$ were nonprofit, rural with $\geq 100$ beds, $11(3.9 \%)$ were not-profit, urban with $<100$ beds, $92(32.4 \%)$ were not-profit, urban with 100 to 299 beds, and $89(31.3 \%)$ were not-profit, urban with $\geq 300$ beds.

The average injurious fall rate for patients aged 65 years or older was $1.28 / 1000$ discharges $(\mathrm{SD}=1.19$, minimum $=0$, maximum $=9.50$ ). Among all 284 hospitals, fully implemented functionality in general medical/surgical units was achieved for the following: electronic clinical documentation in 148 hospitals (52.1\%), results viewing in 255 (89.8\%), CPOE in $63(22.2 \%)$, and decision support in $60(21.1 \%)$.

\subsection{Preliminary Analyses on All Hospitals}

As an exploratory study, preliminary analyses were conducted. We performed 4 independent tests on all 284 included hospitals. No statistically significant findings were seen in the hospital-acquired injurious fall rates among patients aged 65 years or older between hospitals with any 1 of the 4 fully implemented computerized functionalities and those without.

We also performed Pearson $\chi^{2}$ tests to explore the relationship between teaching status $(1=$ yes, $0=$ no $)$ and having each of the 4 fully implemented computerized functionalities. For interpretation purposes, the coding of these 4 measures was recoded as $1=$ yes or $0=$ no. The $\chi^{2}$ test for independence (with Yates continuity correction) indicated a significant association between teaching status and having CPOE in place, $\chi^{2}(1, n=278)=5.07$, $P=0.02$, phi $=0.14$. No significant findings were found for the other 3 functionalities. Therefore, the rest of the tests were performed by teaching status.

\subsection{Results of Testing Research Hypotheses}

Independent $t$ tests were conducted to compare the hospital-acquired injurious fall rates for teaching hospitals with and without each of the 4 functionalities. Table 1 summarizes the findings. No significant difference was found in the hospital-acquired injurious fall rates between the teaching hospitals with and without each of the 4 functionalities.

Independent $t$ tests were also conducted to compare the hospital-acquired injurious fall rates for non-teaching 
hospitals with and without each of the 4 functionalities. Table 2 summarizes the findings. A significant difference was found in the hospital-acquired injurious fall rates between the non-teaching hospitals with $(M=1.03, S D=$
$0.75)$ and without $(M=1.33, S D=1.18)$ electronic clinical documentation $(t=-2.11, P=0.04)$. There was a significant difference in the hospital-acquired injurious fall rates between the non-teaching hospitals with $(M=$

Table 1. Teaching hospitals in the general medical/surgical units: The result summary of the independent $t$ tests on the means of the hospital-acquired injurious fall rates among patients aged 65 years or older between the hospitals with fully implemented functionalities and those without.

\begin{tabular}{|c|c|c|c|c|c|}
\hline Functionality/dependent variable & Status of implementation & $n$ & Mean & $\mathrm{SD}$ & $t$ test value (significance) \\
\hline \multicolumn{6}{|c|}{ Functionality: Electronic clinical documentation } \\
\hline \multirow{2}{*}{ Injurious fall rates per 1000 discharges } & Fully implemented & 45 & 1.68 & 1.42 & \multirow{2}{*}{$t=0.87(P=0.39)$} \\
\hline & Not fully implemented & 43 & 1.39 & 1.62 & \\
\hline \multicolumn{6}{|c|}{ Functionality: Results viewing } \\
\hline \multirow{2}{*}{ Injurious fall rates per 1000 discharges } & Fully implemented & 85 & 1.52 & 1.52 & \multirow{2}{*}{$t=0.01(P=0.99)$} \\
\hline & Not fully implemented & 4 & 1.51 & 1.69 & \\
\hline \multicolumn{6}{|c|}{ Functionality: Computerized provider order entry } \\
\hline \multirow{2}{*}{ Injurious fall rates per 1000 discharges } & Fully implemented & 28 & 1.63 & 1.55 & \multirow{2}{*}{$t=0.48(P=0.64)$} \\
\hline & Not fully implemented & 61 & 1.47 & 1.51 & \\
\hline \multicolumn{6}{|c|}{ Functionality: Decision support } \\
\hline \multirow{2}{*}{ Injurious fall rates per 1000 discharges } & Fully implemented & 25 & 1.67 & 1.47 & \multirow{2}{*}{$t=0.71(P=0.48)$} \\
\hline & Not fully implemented & 61 & 1.42 & 1.52 & \\
\hline
\end{tabular}

Table 2. Non-teaching hospitals in the general medical/surgical units: The result summary of the independent $t$ tests on the means of the hospital-acquired injurious fall rates among patients aged 65 years or older between the hospitals with fully implemented functionalities and those without.

\begin{tabular}{|c|c|c|c|c|c|}
\hline Functionality/dependent variable & Status of implementation & $n$ & Mean & SD & $t$ test value (significance) \\
\hline \multicolumn{6}{|c|}{ Functionality: Electronic clinical documentation } \\
\hline \multirow{2}{*}{ Injurious fall rates per 1000 discharges } & Fully implemented & 103 & 1.03 & 0.75 & \multirow{2}{*}{$t=-2.11^{*}(P=0.04)$} \\
\hline & Not fully implemented & 92 & 1.33 & 1.18 & \\
\hline \multicolumn{6}{|c|}{ Functionality: Results viewing } \\
\hline \multirow{2}{*}{ Injurious fall rates per 1000 discharges } & Fully implemented & 170 & 1.10 & 0.95 & \multirow{2}{*}{$t=-2.38^{*}(P=0.02)$} \\
\hline & Not fully implemented & 24 & 1.61 & 1.14 & \\
\hline \multicolumn{6}{|c|}{ Functionality: Computerized provider order entry } \\
\hline \multirow{2}{*}{ Injurious fall rates per 1000 discharges } & Fully implemented & 35 & 1.24 & 0.94 & \multirow{2}{*}{$t=0.58(P=0.57)$} \\
\hline & Not fully implemented & 154 & 1.13 & 0.99 & \\
\hline \multicolumn{6}{|c|}{ Functionality: Decision support } \\
\hline \multirow{2}{*}{ Injurious fall rates per 1000 discharges } & Fully implemented & 35 & 1.10 & 0.62 & \multirow{2}{*}{$t=-0.56(P=0.58)$} \\
\hline & Not fully implemented & 149 & 1.17 & 1.06 & \\
\hline
\end{tabular}

${ }^{*} \mathrm{P}<0.05$. 
$1.10, S D=0.95)$ and without $(M=1.61, S D=1.14)$ results viewing $(t=-2.38, P=0.02)$. However, no significant difference was found in the hospital-acquired injurious fall rates between the non-teaching hospitals with and without $\mathrm{CPOE}$ and decision support.

\section{DISCUSSION}

\subsection{Limitations of the Study}

This study used publicly available data sources for analysis. Only hospital-level data were used for the analyses. The results could be driven by self-selection (hospitals) into the AHA IT survey supplement. Further, only the 2007 SID data from 3 states were used. For the power analysis for independent $t$ tests, to detect an effect size of 0.30 , a sample of 278 analyzable subjects would provide $80 \%$ power to discover a significant difference at the 0.05 level (independent $t$ tests) $[18,19]$. This study had limited power because only 284 hospitals met the inclusion criteria and only 89 teaching hospitals and 195 non-teaching hospitals were included in the analysis. Although many other determinants in the hospitals could be associated with decreased injurious fall rates, this paper did not obtain information about those other risk factors. Also, no data were available to assess the clinicians' use of each one of the functionalities and the effect of the patterns of that use on patient outcomes.

\subsection{Discussion on the Findings}

Independent $t$ tests were used to answer the research question and test the 4 research hypotheses. The results showed that both the first and second research hypotheses were supported, but only for the non-teaching hospitals. The third and fourth research hypotheses were not supported for either teaching or non-teaching hospitals. In other words, the findings suggested that if a nonteaching hospital has fully implemented the electronic clinical documentation system and the result viewing system in its medical/surgical units, its injurious fall rate would be lower. These 2 specific health IT functionalities were associated with reduced prevalence of injurious falls among adult patients aged 65 years or older in the general medical/surgical units of their hospitals. However, this conclusion was not upheld among teaching hospitals. In short, the significance of this study is that the impact of health IT on the quality of care among the patient group aged 65 years or older was found to vary between teaching and non-teaching hospitals.

Why difference on the hospital-acquired injurious fall rates was identified in the non-teaching but not teaching hospitals in this study? The study conducted by Jha and associates [12] on the use of electronic health records in US hospitals found that larger hospitals, especially those located in urban areas, and teaching hospitals were more likely to have electronic health record systems. Capital requirements and high maintenance costs have been identified as the primary barriers to implementation. The other study conducted by Jha and associates [20] on the progress of adopting electronic health record systems in US hospitals also found that small, public, and rural hospitals were less likely to embrace electronic records than their larger, private, and urban counterparts. It is likely that non-teaching hospitals often have limited resources to be used on quality improvement. When the top executives of a non-teaching hospital committed to adopt electronic health records or selected health IT functionalities (e.g., the electronic clinical documentation system and the result viewing system), capital requirements and the associated maintenance costs were warranted. This determination and movement from the hospital leadership could lead to a change in a hospital's safety culture by putting patient safety first. In contrast, a similar change (e.g., adopting the electronic clinical documentation system and the result viewing system) may not result in a significant change in a teaching hospital's safety culture due to a smaller fiscal impact on teaching hospitals than on non-teaching hospitals. This difference between teaching and non-teaching hospitals may be the explanation why difference on the hospital-acquired injurious fall rates was identified in the non-teaching but not teaching hospitals. This argument needs to be verified in future research.

\subsection{Practical Implications}

It is noted that in the current acute care hospital settings, about $75 \%$ of the inpatients aged 65 or older (estimated). Therefore, promoting patient information exchange in an effective and timely manner should be a focus of health IT functionality upgrades to improve nursing care outcomes in non-teaching hospitals, specific to preventing hospital-acquired injurious fall incidents among patients aged 65 or older. Implementing the electronic clinical documentation and result viewing systems in the medi$\mathrm{cal} /$ surgical units of non-teaching hospitals could inform nursing staff of best practice interventions through achieving a shared goal of displaying and exchanging patient information in an effective and timely manner $[7,8]$.

\section{CONCLUSION}

Shifting limited hospital resources, which are used for interventions to prevent adverse events (e.g., hospital-acquired injurious falls), could create unanticipated consequences. One consequence for interventions would be fewer lawsuits for liability and lower costs of liability insurance for hospitals, as well as fewer expenses for longer stays due to hospital-acquired injuries resulting 
from falls. It is recognized that a margin of diminishing returns exists where an intervention (the burden to each hospital) would cost too much to prevent a highly unlikely risk (the probability) for falling and acquiring severe injuries (the harm), unless the cost of each fall is large. A low probability of a large magnitude of harm may still be cost effective based on the intervention (e.g., the IT functionalities of electronic clinical documentation and results viewing). Future research may focus on assessing the clinicians' use of the IT functionalities of electronic clinical documentation and results viewing, as well as the effect of the clinicians' use patterns on patient outcomes.

\section{ACKNOWLEDGEMENTS}

This project was supported by Bryer Research Fund Grant, University of Michigan-Flint, Flint, Michigan, USA. The authors also would like to thank Dr. Terry Van Allen for his support and assistance. The authors declare that they have no competing interests.

\section{REFERENCES}

[1] Oliver, D., Healey, F. and Haines, T.P. (2010) Preventing falls and fall-related injuries in hospitals. Clinics in Geriatric Medicine, 26, 645-692.

doi:10.1016/j.cger.2010.06.005

[2] McGowan, J.J. and Roper, R.A. (2010) The Health IT Value Grant Initiative: A programmatic review of the peer-reviewed literature. AHRQ Publication No. 100063-EF. Agency for Healthcare Research and Quality, Rockville.

[3] Dykes, P.C., Carroll, D.L., Hurley, A., Lipsitz, S., Benoit, A., Chang, F., Meltzer, S., Tsurikova, R., Zuyov, L. and Middleton, B. (2010) Fall prevention in acute care hospitals: a randomized trial. Journal of the American Medical Association, 304, 1912-1918. doi:10.1001/jama.2010.1567

[4] Tzeng, H.M., Hu, H.M. and Yin, C.Y. (2011) Is there a link between the hospital-acquired injurious fall rates in US acute care hospitals and these institutions' implementation levels of computerized systems? CIN: Computers, Informatics, Nursing, 29, 721-729.

[5] Centers for Medicare \& Medicaid Services (CMS) (2008) Changes to the hospital inpatient prospective payment systems and fiscal year 2009 rates. http://www.cms.hhs.gov/AcuteInpatientPPS/downloads/C MS-1390-F.pdf

[6] Healthcare Cost and Utilization Project (2011) Overview of the state inpatient databases (SID). http://www.hcup-us.ahrq.gov/sidoverview.jsp\#Data

[7] American Hospital Association (AHA) (2008) Hospital EHR adoption database ${ }^{\mathrm{TM}}$.

http://www.ahadata.com/ahadata/html/EHRdatabase.html

[8] American Hospital Association (AHA) (2008) 2007 AHA annual survey-Information technology supplement. http://www.ahadata.com/ahadata/files/2009/IT-Suppleme

\section{ntSurvey.pdf}

[9] Library of Congress (2010) The one hundred eleventh congress of the United States of America at the second section: The patient protection and affordable care act $(\mathrm{H}$. R. 3590). US Government Printing Office, Washington DC.

http://www.gpo.gov/fdsys/pkg/BILLS-111hr3590enr/pdf/ BILLS-111hr3590enr.pdf

[10] Stead, W.W. and Herbert, S.L. (2009) Summary. In: Stead, W.W. and Herbert, S.L. Eds., Computational Technology for Effective Health Care: Immediate Steps and Strategic Directions. The National Academy of Sciences, Washington DC, S10-S11.

[11] Law, B. (2010) The Health Information Technology for Economic and Clinical Health Act (HITECH) Act. http://lawbrain.com/wiki/Health_Information_Technolog y_for_Economic and_Clinical_Health_Act_(HITECH)

[12] Jha, A.K., DesRoches, C.M., Campbell, E.G., Donelan, K., Rao, S.R., Ferris, T.G., Shields, A., Rosenbaum, S. and Blumenthal, D. (2009) Use of electronic health records in U.S. hospitals. New England Journal of Medicine, 360, 1628-1638. doi:10.1056/NEJMsa0900592

[13] National Quality Forum (NQF) (2010) Safe practices for better healthcare-2010 update: A consensus report. National Quality Forum, Washington DC.

[14] Coffey, R., Milenkovic, M. and Andrews, R.M. (2006) HCUP methods series report \# 2006-01: The case for the present-on-admission (PoA) indicator. http://www.hcup-us.ahrq.gov/reports/2006_1.pdf

[15] Csikesz, N.G., Singla, A., Simons, J.P., Tseng, J.F. and Shah, S.A. (2009) The impact of socioeconomic status on presentation and treatment of diverticular disease. Journal of Gastrointestinal Surgery, 13, 1993-2001. doi:10.1007/s11605-009-1031-3

[16] Dartmouth Atlas of Health Care (2007) Medicare reimbursements per enrollee, by gender and program component.

http://www.dartmouthatlas.org/data/table.aspx?ind $=123 \&$ loct $=3 \& \mathrm{tf}=10 \& \mathrm{ch}=32,19$

[17] American Hospital Association (AHA) (2008) AHA annual survey database for fiscal year 2007. Health Forum LLC, Chicago.

[18] Faul, F., Erdfelder, E., Buchner, A. and Lang, A.G. (2009) Statistical power analyses using G*Power 3.1: Tests for correlation and regression analyses. Behavior Research Methods, 41, 1149-1160. doi:10.3758/BRM.41.4.1149

[19] Faul, F., Erdfelder, E., Lang, A.G. and Buchner, A. (2007) $G^{*}$ Power 3: A flexible statistical power analysis program for the social, behavioral, and biomedical sciences. Behavior Research Methods, 39, 175-191. doi:10.3758/BF03193146

[20] Jha, A.K., DesRoches, C.M., Kralovec, P.D. and Joshi, M.S. (2010) A progress report on electronic health records in US hospitals. Health Affairs (Millwood), 29, 1951-1957. doi:10.1377/hlthaff.2010.0502 